\title{
Gamification in a Physical Rehabilitation Setting: Developing a Proprioceptive Training Exercise for a Wrist Robot
}

\author{
Christopher \\ Curry \\ University of \\ Minnesota \\ curry134@umn.edu
}

\author{
Naveen \\ Elangovan \\ University of \\ Minnesota \\ naveen@umn.edu
}

\author{
Reuben Gardos \\ Reid \\ University of \\ Minnesota \\ gardo007@umn.edu
}

\author{
Jiapeng Xu \\ University of \\ Minnesota \\ xu000219@umn.edu
}

\author{
Jürgen Konczak \\ School of Kinesiology \\ University of \\ Minnesota \\ jkonczak@umn.edu
}

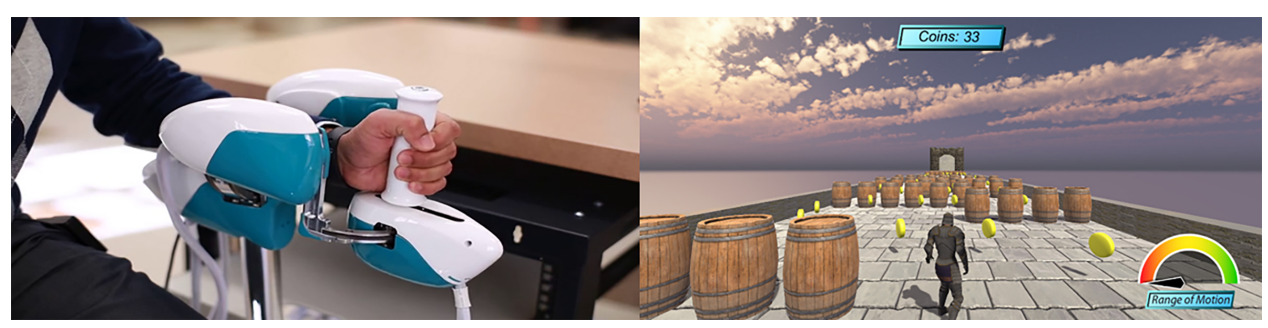

Figure 1: Left. WristBot being used by a participant. Right. Screenshot of the virtual environment showing an avatar controlled by user's wrist movements.

\begin{abstract}
Proprioception or body awareness is an essential sense that aids in the neural control of movement. Proprioceptive impairments are commonly found in people with neurological conditions such as stroke and Parkinson's disease. Such impairments are known to impact the patient's quality of life. Robot-aided proprioceptive training has been proposed and tested to improve sensorimotor performance. However, such robot-aided exercises are implemented similar to many physical rehabilitation exercises, requiring taskspecific and repetitive movements from patients. Monotonous nature of such repetitive exercises can result in reduced patient motivation, thereby, impacting treatment adherence and therapy gains. Gamification of exercises can make physical rehabilitation more engaging and rewarding. In this work, we discuss our ongoing efforts to develop a game that can accompany a robot-aided wrist proprioceptive training exercise.
\end{abstract}

\section{CCS CONCEPTS}

- Human-centered computing $\rightarrow$ Virtual reality; User Studies.

\section{KEYWORDS}

Gamification, Proprioception, Proprioception Training, Wristbot

\section{ACM Reference Format:}

Christopher Curry, Naveen Elangovan, Reuben Gardos Reid, Jiapeng Xu, and Jürgen Konczak. 2019. Gamification in a Physical Rehabilitation Setting:

Permission to make digital or hard copies of part or all of this work for personal or classroom use is granted without fee provided that copies are not made or distributed for profit or commercial advantage and that copies bear this notice and the full citation on the first page. Copyrights for third-party components of this work must be honored.

For all other uses, contact the owner/author(s).

SA '19 Posters, November 17-20, 2019, Brisbane, QLD, Australia

(C) 2019 Copyright held by the owner/author(s).

ACM ISBN 978-1-4503-6943-5/19/11.

https://doi.org/10.1145/3355056.3364572
Developing a Proprioceptive Training Exercise for a Wrist Robot. In SIGGRAPH Asia 2019 Posters (SA '19 Posters), November 17-20, 2019, Brisbane, QLD, Australia. ACM, New York, NY, USA, 2 pages. https://doi.org/10.1145/ 3355056.3364572

\section{INTRODUCTION}

Proprioception, the sense of body awareness, is essential for normal motor function. Proprioceptive deficits are common in neurological conditions [Coupar et al. 2012; Konczak et al. 2009]. Such deficits cause a decline in precision of goal-directed movements, and altered postural and spinal reflexes resulting in balance and gait problems [Rothwell et al. 1982]. Proprioceptive training is an intervention aiming to improve proprioceptive function [Aman et al. 2015]. Previous work has established the efficacy of a robot-aided proprioceptive training using WristBot [Elangovan et al. 2017, 2018, 2019]. The WristBot (Figure 1. Left) is a three degrees-of-freedom (3-DoF) exoskeleton robot that allows full range of motion (ROM), delivers precise haptic, position, and velocity stimuli at the wrist, and accurately encodes wrist position across time. Additional details about the WristBot can found in [Cappello et al. 2015].

Nevertheless, while the WristBot has demonstrated its efficacy, it shares a limitation that is often encountered in rehabilitation settings. In a clinical setting, patients are often required to perform task-specific and repetitive movements [Kwakkel et al. 1999]. Initial patient enthusiasm to complete such activities rapidly declines as a result of the monotonous nature of movements. Patient engagement can be improved by complementing therapy with a virtual environment (VE). Prior research has shown that users have favored exercises complemented with a VE rather than conventional approaches [Hoffman et al. 2014]. Thus, our project objective is to turn these tedious movements into an interactive VE experience. 


\section{GAMIFICATION OF PROPRIOCEPTIVE TRAINING}

Gamification process accounted for two key considerations: (1) the game should foster patient motivation and attention (2) and be clinically meaningful. To address these objectives, we reviewed the literature on game development [Bond 2014; Fullerton 2018] and identified four essential components: (1) Variability, (2) Feedback, (3) Rewards, and (4) a Compelling Purpose. The user will be gradually exposed to increasing levels of difficulty, which will likely reduce user frustrations. The user will receive meaningful feedback on concurrent metrics (e.g., Optimal ROM), as well as on previous treatment sessions. During game progress, the user will be alerted about deviations from the target movement requirements. Achievement badges will be rewarded to the user upon reaching therapy milestones, such as target ROM. Lastly, to encourage game completion, we establish an interesting backstory and a meaningful character arc for our virtual avatars. The developed game will be adaptable based on the user's current clinical status, thus, making the game clinically meaningful. The clinician will have the ability to prescribe exercises based on user needs such as 1 DoF vs 3 DoF movements, continuous vs discrete movements, and strength training vs mobility training. WristBot will provide supportive forces aiding the user to achieve therapy milestones.

Gamified exercise is being developed using the Unity Game Engine, Python and libraries which interface with the WristBot. The game closely resembles an endless runner type game (Figure 1. Right) and utilizes the WrsitBot's 3-DoF functionality to interact with the VE. Wrist flexion, extension, and abduction can be used to traverse their environment. The remaining 3 movements will allow interactions with their VE in unique ways, such as opening/closing doors, crouching, and pulling levers. In the VE, coins are strategically placed to maximize and improve the use of available ROM. Upon contact with either a wall or obstacle, visual feedback will be provided in the form of avatar damage and coin deduction. Consequently, users achieve improved mobility.

In Python, the connection between Unity and the WristBot library is managed through the use of a local WebSocket, a protocol for two-way communication over a single Transmission Control Protocol (TCP) connection [Fette and Melnikov 2011]. Through the WebSocket, reciprocal data are transferred between the WristBot and Unity. For example, wrist kinematic data will be streamed to the game while game progress is being relayed to the WristBot library. Game progress data will be utilized to compute and deliver haptic feedback to the user. Haptic feedback provided in the form of haptic assistance will aid users to improve their available ROM, while haptic resistance will improve muscle strength within the desired ROM. The clinical motive of the game is to transition the user from use of haptic assistance to resistance during game play. WristBot will adapt haptic feedback based on time spent and progress achieved in game play.

\section{USABILITY TESTING}

Usability testing will be conducted to ensure proper game usage by the clinical population and healthcare professionals. Specifically, the usability testing will evaluate areas such as 1) ease of game play, 2) game efficiency, and 3) user engagement. We will test the assumptions in each of these areas are accurately depicted in game development and met during game play. For example, we expect online visual feedback of deviations from target to help user focus on achieving the movement requirements. The users will be asked to verify the benefits of visual feedback in modifying their movements. Similarly, other assumptions such as performance badges and coins as rewards, and increase in difficulty levels will be evaluated. A common pitfall of usability studies involving physical rehabilitation setting is not recruiting from the representative population, most notably elderly population [Laver et al. 2017] as age has been shown to interfere with interactions in VE [Meldrum et al. 2012]. Therefore, to ensure our game is intuitive, we will recruit representative users from our patient populations.

\section{ACKNOWLEDGMENTS}

This project was supported by National Science Foundation Partnerships For Innovation Technology Translation Award to Jürgen Konczak (1919036). Christopher Curry was supported by National Research Trainee-Understanding the Brain: Graduate Training Program in Sensory Science: Optimizing the Information Available for Mind and Brain (1734815).

\section{REFERENCES}

Joshua E Aman, Naveen Elangovan, I Yeh, Jürgen Konczak, et al. 2015. The effectiveness of proprioceptive training for improving motor function: a systematic review. Frontiers in human neuroscience 8 (2015), 1075

Jeremy Gibson Bond. 2014. Introduction to Game Design, Prototyping, and Development: From Concept to Playable Game with Unity and C. Addison-Wesley Professional.

Leonardo Cappello, Naveen Elangovan, Sara Contu, Sanaz Khosravani, Jürgen Konczak, and Lorenzo Masia. 2015. Robot-aided assessment of wrist proprioception. Frontiers in human neuroscience 9 (2015), 198.

Fiona Coupar, Alex Pollock, Phil Rowe, Christopher Weir, and Peter Langhorne. 2012. Predictors of upper limb recovery after stroke: a systematic review and metaanalysis. Clinical rehabilitation 26, 4 (2012), 291-313.

Naveen Elangovan, Leonardo Cappello, Lorenzo Masia, Joshua Aman, and Jürgen Konczak. 2017. A robot-aided visuo-motor training that improves proprioception and spatial accuracy of untrained movement. Scientific reports 7, 1 (2017), 17054.

Naveen Elangovan, Paul Tuite, and Jürgen Konczak. 2018. Somatosensory training improves proprioception and untrained motor function in Parkinson's disease. Frontiers in neurology 9 (2018), 1053.

Naveen Elangovan, I-Ling Yeh, Jessica Holst-Wolf, and Jürgen Konczak. 2019. A robot-assisted sensorimotor training program can improve proprioception and motor function in stroke survivors. In 2019 IEEE 16th International Conference on Rehabilitation Robotics (ICORR). IEEE, 660-664.

I. Fette and A. Melnikov. 2011. The WebSocket Protocol. Technical Report

Tracy Fullerton. 2018. Game design workshop: a playcentric approach to creating innovative games. AK Peters/CRC Press.

Hunter G Hoffman, Walter J Meyer III, Maribel Ramirez, Linda Roberts, Eric J Seibel, Barbara Atzori, Sam R Sharar, and David R Patterson. 2014. Feasibility of articulated arm mounted Oculus Rift Virtual Reality goggles for adjunctive pain control during occupational therapy in pediatric burn patients. Cyberpsychology, Behavior, and Social Networking 17, 6 (2014), 397-401.

Jürgen Konczak, Daniel M Corcos, Fay Horak, Howard Poizner, Mark Shapiro, Paul Tuite, Jens Volkmann, and Matthias Maschke. 2009. Proprioception and motor control in Parkinson's disease. Journal of motor behavior 41, 6 (2009), 543-552.

Gert Kwakkel, Boudewijn J Kollen, and Robert C Wagenaar. 1999. Therapy impact on functional recovery in stroke rehabilitation: a critical review of the literature. Physiotherapy 85, 7 (1999), 377-391.

Kate E Laver, Belinda Lange, Stacey George, Judith E Deutsch, Gustavo Saposnik, and Maria Crotty. 2017. Virtual reality for stroke rehabilitation. Cochrane database of systematic reviews 11 (2017).

Dara Meldrum, Aine Glennon, Susan Herdman, Deirdre Murray, and Rory McConnWalsh. 2012. Virtual reality rehabilitation of balance: assessment of the usability of the Nintendo Wii ${ }^{\circledR}$ Fit Plus. Disability and rehabilitation: assistive technology 7, 3 (2012), 205-210.

JC Rothwell, MM Traub, BL Day, JA Obeso, PK Thomas, and CD Marsden. 1982. Manual motor performance in a deafferented man. Brain 105, 3 (1982), 515-542. 